\title{
Mesangial IgM Deposition predicts renal outcome in patients with IgA nephropathy: A multicenter, prospective, observational study
}

\section{Li Tan}

Division of Nephrology, Department of Medicine, West China Hospital, Sichuan University, Chengdu, Sichuan, China. West China School of Medicine, Sichuan University, Chengdu, Sichuan, China

\section{Yi Tang}

Division of Nephrology, Department of Medicine, West China Hospital, Sichuan University, Chengdu,

Sichuan, China

\section{Gai-Qin Pei}

Division of Nephrology, Department of Medicine, West China Hospital, Sichuan University, Chengdu, Sichuan, China. West China School of Medicine, Sichuan University, Chengdu, Sichuan, China

\section{Zheng-Xia Zhong}

Division of Nephrology, Department of Medicine, Affiliated Hospital of Zunyi Medical University, Medical University, Zunyi, Guizhou, China. West China School of Medicine, Sichuan University, Chengdu, Sichuan, China

\section{Jia-Xing Tan}

Division of Nephrology, Department of Medicine, West China Hospital, Sichuan University, Chengdu, Sichuan, China. West China School of Medicine, Sichuan University, Chengdu, Sichuan, China

\section{$\mathrm{Ya} \mathrm{Ma}$}

Department of Clinical Nutrition, West China Hospital, Sichuan University, Chengdu, Sichuan, China

\section{Dong-Guang Wang}

Department of Respiratory and Critical Care Medicine, West China Hospital/ Sichuan University, Chengdu, China. West China School of Medicine, Sichuan University, Chengdu, Sichuan, China

\section{Ling Zhou}

Division of Nephrology, Zigong Third People's Hospital, Zigong, Sichuan, China. West China School of Medicine, Sichuan University, Chengdu, Sichuan, China

\section{David Sheikh-Hamad}

Section of Nephrology, Department of Medicine, Baylor College of Medicine

\section{Wei Qin ( $\nabla$ qinweihx@scu.edu.cn )}

Sichuan University West China Hospital https://orcid.org/0000-0003-4246-5431

\section{Research}


Keywords: Immunoglobulin A nephropathy; IgM deposition》renal outcomes囚clinicopathological features; propensity score matching (PSM)

Posted Date: May 18th, 2020

DOI: https://doi.org/10.21203/rs.3.rs-28079/v1

License: (c) (i) This work is licensed under a Creative Commons Attribution 4.0 International License. Read Full License

Version of Record: A version of this preprint was published at Clinical and Experimental Medicine on April 10th, 2021. See the published version at https://doi.org/10.1007/s10238-021-00703-1. 


\section{Abstract}

\section{Background}

Mesangial IgM deposition is commonly found in patients with immunoglobulin A nephropathy (IgAN). This study aims to investigate the relationships between mesangial $\lg \mathrm{M}$ deposition and disease progression in IgAN patients.

\section{Methods}

A total of 1239 patients with biopsy-proven primary IgAN were enrolled in this multicenter, prospective, observational study between January 2013 and August 2017. According to the degree of IgM deposition, 1239 patients were divided into three groups: Grade 0 (no or trace; $n=713,57.55 \%$ ), Grade 1 (mild; $n=$ 414, 33.41\%), Grades $2+3$ (moderate and marked; $n=112,9.04 \%$ ). Using a 1: 1 propensity score matching (PSM) method identifying age, gender, and treatment modality to minimize confounding factors, 1042 matched patients (out of 1239) with different degrees of IgM deposition were enrolled to evaluate the severity of baseline clinicopathological features and renal outcome: Grade $0(n=521$, $50.00 \%)$, Grade $1(n=409,39.25 \%)$, Grades $2+3(n=112,10.75 \%)$. Kaplan-Meier and Cox proportional hazards analyses were performed to determine whether different degrees of mesangial IgM deposition are associated with varying renal outcomes in IgAN.

Results

During a mean follow-up of $48.90 \pm 23.86$ and $49.01 \pm 23.73$ months, before and after adjusting for propensity scores respectively, the rate of complete remission (CR) was progressively lower with increased IgM deposition in both unmatched $(63.39 \%, 46.14 \%, 45.54 \%)$ and matched cohort $(61.80 \%$, $46.45 \%, 45.54 \%)$, whereas the proportion of patients progressing to end stage renal disease (ESRD) showed reverse correlation $(P<0.001)$. Kaplan-Meier analysis indicated negative correlation between the intensity of mesangial IgM deposits and cumulative renal survival (all $P<0.05$ ). Moreover, Cox regression analysis revealed that the degree of mesangial IgM deposition predicted renal outcome independent of MESTC score and clinical variables in the unmatched (Grade 1, HR, 1.59; 95\% Cl, 1.11-2.29; $P=0.01$; Grades $2+3$, HR, 1.69; 95\% Cl, 1.02-2.08; $P=0.04$ ) and matched cohort (Grade 1, HR, 1.84; 95\% Cl, 1.192.85; $P=0.01 ;$ Grades $2+3, \mathrm{HR}, 1.91 ; 95 \% \mathrm{Cl}, 1.01-3.24 ; P=0.04)$.

\section{Conclusions}

Mesangial lgM deposition plays an important role in renal prognosis and is independently associated with worse renal outcomes in patients with IgAN.

Trial registration TCTR, TCTR20140515001. Registered May 15, 2014, http://www.clinicaltrials.in.th/index.php? tp=regtrials\&menu=trialsearch\&smenu=fulltext\&task=search\&task2=view1\&id=1074 


\section{Introduction}

Immunoglobulin A nephropathy (IgAN) is the most common cause of primary glomerulonephritis (GN) worldwide ${ }^{1}$. The diagnosis of IgAN is based on dominant or co-dominant mesangial deposits of IgA alone or with IgG and/or IgM in the glomerular mesangium ${ }^{1,2}$. Recent studies revealed that mesangial deposition of abnormally glycosylated IgA1 molecule could activate local complement system and induce inflammation via the classical, alternative and lectin pathway ${ }^{3,4}$. IgM deposition is commonly seen in the glomerular mesangial region in approximately $80 \%$ of IgAN patients. However, the clinical significance of mesangial IgM Deposition in IgAN patients has not been completely understood. Considering that IgM deposition could aggravate glomerular injury in animal models of glomerular diseases ${ }^{5,6}$ through complement activation ${ }^{7}$, we hypothesized that IgM deposition in IgAN patients may also be important for disease development. Previously, several studies reported that IgAN patients with glomerular IgM deposition had worse clinical features and renal prognosis than those without ${ }^{8}$, while other studies found no association between IgM deposition and clinical manifestation ${ }^{9}$.

In this multicenter, prospective, observational study, we examined whether mesangial IgM deposition is associated with clinicopathological features or renal outcomes in IgAN patients.

\section{Materials And Methods}

\section{Patients}

A total of 1239 patients with biopsy-proven primary IgAN diagnosed at 4 nephrology centers (West China Hospital, Affiliated Hospital of Zunyi Medical University, Zigong Third People's Hospital and People's Hospital of Mianzhu city) from January 2013 to August 2017 were enrolled in this study. Exclusion criteria were as followed: (i) secondary IgAN due to systemic diseases such as systemic lupus erythematosus, Henoch - Schönlein purpura and liver cirrhosis, etc.; (ii) lupus nephritis; (iii) C1q nephropathy; (iv) pregnancy; (v) hepatitis B virus carriers and other chronic liver diseases; (vi) baseline estimated glomerular filtration rate (eGFR) $<15 \mathrm{ml} / \mathrm{min} / 1.73 \mathrm{~m}^{2}$ or receive renal replacement treatment; (vii) <18-year old before the renal biopsy; (viii) without sufficient clinicopathological information (renal biopsies less than 8 glomeruli, discontinued follow-up or treatment)

Patients were followed up for at least 12 months or shorter if they reached study endpoints. Patients were followed up monthly in study centers. The research complied with the Declaration of Helsinki and was approved by the ethics committees of all study centers. Written informed consent was obtained from each patient.

\section{Clinical data}

Demographics (gender, age, ethnicity) and clinical data (blood pressure (BP), serum albumin (Alb), serum creatinine (Scr), and $24 \mathrm{~h}$ urinary protein at the time of biopsy were collected. The estimated GFR (eGFR) 
was evaluated according to CKD-EPI (CKD Epidemiology Collaboration) formula ${ }^{10}$. Mean arterial pressure (MAP) was defined as diastolic pressure plus a third of the pulse pressure.

\section{Renal histopathology}

All patients were diagnosed as IgAN based on histopathology assessment of biopsy tissue. Kidney specimens obtained by ultrasound-guided percutaneous needle biopsy were embedded in paraffin. Serial sections 2-3 $\mu \mathrm{m}$ thick were evaluated by light microscopy (stained with hematoxylin and eosin (H\&E), periodic acid-Schiff, Masson's trichrome and periodic acid methenamine), immunofluorescence (stained with antibodies against IgA, IgG, IgM, C3, C4, C1q and kappa and lambda chains), and electron microscopy respectively. We set a minimum requirement of 8 glomeruli for light microscopy and three glomeruli for immunofluorescence microscopy. For immunofluorescence, the sections were washed thrice with PBS for 3 min each time, dripped with human IgM monoclonal antibody (1:100; cat nos. ab212201; Abcam, Cambridge, MA, USA), incubated in water bath at $37^{\circ} \mathrm{C}$ for $60 \mathrm{~min}$, and washed thrice with PBS for 3 min each time. The sections were then incubated with Alexa Fluor ${ }^{\circledR} 488$ goat anti-rabbit secondary antibody (1:200; Cat nos. ab150077; Abcam, Cambridge, MA, USA), and imaged using fluorescence microscopes. The degree of IgM deposits was semiquantitatively determined (grade 0 , no or trace; grade 1, mild; grade 2, moderate; grade 3, marked) in the mesangium and along the glomerular capillary loops, respectively.

Renal biopsy specimens of all patients were reviewed by experienced pathologists and nephrologists who were blinded to each other's evaluations as well as patients' clinical data. The discrepancy in diagnosis between pathologists and nephrologists was resolved by reviewing the biopsies and coming to a consensus. Pathological lesions were graded according to the updated Oxford classification ${ }^{11}$ : mesangial hypercellularity (M0/M1); endocapillary hypercellularity (E0/E1); segmental glomerulosclerosis (S0/S1); tubular atrophy / interstitial fibrosis (T0/T1/T2) ${ }^{11}$ and cellular or fibrocellular crescents (C0/C1/C2) ${ }^{12}$.

\section{Treatment}

Treatment modalities were recorded. Patients were treated with 3 different treatment strategies as described previously ${ }^{13}$ : supportive care (SC, full dose angiotensin-converting-enzyme inhibitor or angiotensin receptor blockers), supportive care combined with corticosteroids (CS, $0.5-1 \mathrm{mg} / \mathrm{kg}$ prednisone daily, tapering down within 6 - 8 months) and supportive care plus corticosteroids and immunosuppressant therapy (IT, cyclophosphamide $2 \mathrm{mg} / \mathrm{kg}$ daily for 3 months, or MMF 1-2g daily for 68 months, or cyclosporine $3-5 \mathrm{mg} / \mathrm{kg}$ daily for $6-8$ months, or tacrolimus $0.03-0.05 \mathrm{mg} / \mathrm{kg}$ daily for 6-8 months).

\section{Treatment Responses}

Responses to therapy included complete remission (CR), partial remission (PR), no response (NR) and end-stage renal disease (ESRD). CR was defined as urinary protein excretion $<0.5 \mathrm{~g} / 24 \mathrm{~h}$, with eGFR 
decrease $<10 \%$ baseline. PR was defined as proteinuria decrease $>50 \%$ baseline, with eGFR decrease $<$ $10 \%$ baseline. NR was defined as proteinuria decrease $<50 \%$ baseline, or eGFR decrease $>10 \%$ baseline. ESRD was defined as eGFR $<15 \mathrm{~mL} / \mathrm{min} / 1.73 \mathrm{~m}^{2}$ or maintenance renal replacement treatment. For evaluation of renal outcomes, the primary endpoint was the combined endpoint of a $50 \%$ decline of eGFR and/or ESRD or death. Renal survival time was the time point when patients reached the primary endpoint or the last visit if the patients did not reach the primary endpoint during the entire follow-up period.

\section{Statistical Analysis}

All statistical analyses were performed by using $\mathrm{R}$ version 3.3.4 statistical software (The R Foundation for Statistical Computing) and GraphPad Prism7.0. Continuous variables were analyzed with ANOVA, Kruskal-Wallis $\mathrm{H}$ test, Student's $\mathrm{t}$ test or nonparametric Mann-Whitney $\mathrm{U}$ test and presented as mean \pm standard deviation (SD) or median [interquartile range (IQR)]. Categorical data were analyzed using Chisquare tests or Fischer's exact test and presented as frequencies (percentages). Risk factors for IgAN patients were analyzed using the Cox regression model. Results were expressed as hazard ratios (HRs) and their $95 \%$ confidence intervals (Cls). Kaplan-Meier curves were used to analyze the outcomes of patients. Statistical significance was determined as $p<0.05$.

In this study, all patients were assigned a 1: 1 propensity score matching (PSM) identifying age, gender, and treatment modality with the occurrence of IgM deposition as the dependent variable to adjust for the observed characteristics of non-randomly assigned patients. PSM is a statistical matching technique that attempts to estimate the effectiveness of treatments, policies, or other interventions by taking covariates into account ${ }^{14,15}$. PSM was derived from a multi-logistic regression model containing age, gender, and treatment modality to reduce the bias. The propensity score (PS) is calculated by the following conditional probability.

$$
\mathrm{p}\left(x_{i}\right)=\operatorname{pr}\left(\mathrm{T}=1 \mid X=x_{i}\right)=\frac{\exp \left(r_{0}+r_{1} \text { sex }_{i}+r_{2} a g e_{i}+r_{3} B M I_{i}\right)}{\exp \left(r_{0}+r_{1} \text { Sex }_{i}+r_{2} a g e_{i}+r_{3} B M I_{i}\right)+1}
$$

The caliper is defined by the maximum propensity score difference within the matched pair. Three methods of matching individuals with similar propensity scores are presented based on the concept of the caliper in the R package Matchlt ${ }^{16}$. Since it was necessary to ensure balanced matches among the Grade 0 , Grade 1, Grades $2+3$ groups, the caliper (from 0 to 1 ) was manipulated by increments of 0.01 . To ensure balanced matches, a caliper and maximum allowable difference among three groups were defined as 0.1 , resulting in a relatively narrow difference among matched subjects ${ }^{15}$. Standardized mean differences were estimated for all baseline covariates before and after matching to assess pre-match imbalance and post-match balance.

\section{Results}




\section{Demographic and Clinicopathological Features of Patients}

1494 IgAN patients from 4 study centers were recruited. In total, 255 patients were excluded and 1239 patients were enrolled (Fig.1). Demographic and clinicopathological characteristics of the patients at the time of biopsy are shown in Table 1. The average follow-up period was $48.90 \pm 23.86$ and $49.01 \pm 23.73$ months before and after adjustment (unmatched and matched cohort) for propensity scores, respectively. In the unmatched cohort, 1239 patients were categorized into 3 groups according to the degree of glomerular IgM deposition in glomeruli: Grade 0 (713, 57.55\%); Grade 1 (414, 33.41\%); Grades $2+3$ (112, $9.04 \%)$. Of note, more female and younger patients were found among the patients with a high level of IgM deposition in the unmatched cohort. After 1: 1 PSM identifying age, gender, and treatment modality, a total of 1042 patients were enrolled in the matched study: 521 (50.00\%) in Grade 0, $409(39.25 \%)$ in Grade 1, 112 (10.75\%) in Grades 2+3 groups, respectively (Table 1). Both in the unmatched and matched cohort, patients with a higher level of IgM deposits presented with worse clinical and pathological features, such as higher proteinuria, lower serum albumin, higher proportions of M1, E1 and C1/C2 lesions and more glomerular deposits of IgG, C3, C4 and C1q. Moreover, patients with IgM deposition were treated much more aggressively (with more corticosteroids plus immunosuppressant therapy) (Table 1).

\section{Comparison of Clinical Parameters at the time of Kidney Biopsy and Decline Rate of Renal Function according to the Oxford Classification and Glomerular IgM Deposits}

The baseline distribution of the pathological variables and the comparison of clinical data according to the Oxford-MESTC classification and the degrees of glomerular IgM deposits are shown in Table 2. At the time of biopsy, a higher level of baseline proteinuria was present in the unmatched and matched patients with E1, T1/T2, C1/C2 lesion and higher intensity of IgM and C1q glomerular deposits. Furthermore, patients with higher MESTC lesion scores on the Oxford classification and higher intensity of glomerular C1q deposits had a significantly lower level of baseline eGFR in the unmatched and matched cohorts. The rate of decline in eGFR was greater in patients with T1/T2 compared with $\mathrm{M} 0$ both in the unmatched and matched patients. Moreover, a multivariate linear regression analysis was used to identify pathological variables associated with a decline in renal function. After adjustment for hypertension, initial eGFR and proteinuria, the following correlated with faster rate of eGFR decline: T1/T2 $(\beta=-4.77$, $P=0.002)$ in the unmatched cohort; T1/T2 $(\beta=-4.60, P=0.04)$ and glomerular $\mathrm{C} 4$ deposits $(\beta=6.39$, $P=0.04$ ) in the matched cohort (Table 2). However, there were no differences in baseline eGFR and the rate of decline in eGFR according to glomerular IgM deposits.

\section{Treatment Responses and Renal Outcomes}

The treatment responses of IgAN patients are shown in Table 3. After treatment, 862 (69.57\%) patients obtained remission, including CR in 694 (56.01\%) and PR in 168 (13.56\%); 94 (7.59\%) patients progressed to $\operatorname{ESRD}(P<0.001)$. Of note, patients with IgM deposition had a lower rate of remission and higher rate of ESRD both in the matched and unmatched cohort. 
Only 1 patient with apparent IgM deposition (Grade 3) died of pneumonia after 2 months of corticosteroids treatment. In terms of the primary endpoint of a $50 \%$ decline of eGFR and/or ESRD or death, patients with a higher degree of glomerular IgM deposition presented a significantly higher incidence of endpoint events in both the unmatched and matched cohort (Table 4). With the combined endpoint of a 50\% decline in eGFR and/or ESRD, Kaplan- Meier analysis indicated that cumulative renal survival was significantly lower in patients with a higher degree of glomerular IgM deposits both in the unmatched $(P=0.03)$ and matched cohort $(P=0.02)$ (Fig.2).

\section{Renal failure predictors}

In the univariate Cox regression analysis, glomerular deposition of IgM was identified as one of the risk factors for poor renal outcome in IgAN patients in both the unmatched and matched cohort (Table 5, model 1). In a multivariate Cox proportional model, the combined endpoint was significantly associated with hypertension, low eGFR, urinary protein and degree of glomerular IgM deposits (model 2). In the final model (model 3 ) in which glomerular IgM deposits was adjusted for hypertension, eGFR, proteinuria, Oxford renal pathological classification (MESTC), the degree of glomerular IgM deposits was a significant predictor of poor renal outcome in the unmatched (Grade 1, HR, 1.59; 95\% Cl, 1.11-2.29; $P=0.01$; Grades 2+3, HR, 1.69; 95\% Cl, 1.02-2.08; $P=0.04$ ) and matched cohort (Grade 1, HR, 1.84; $95 \% \mathrm{Cl}$, 1.19-2.85; $P=0.01$; Grades 2+3, $\mathrm{HR}, 1.91 ; 95 \% \mathrm{Cl}, 1.01-3.24 ; P=0.04)$.

\section{Discussion}

Although the clinical presentation and course of IgAN are quite variable, $20-40 \%$ of the patients progress to ESRD within 10-20 years from the onset of the disease ${ }^{1,17}$. IgM deposition is commonly seen in the glomerular mesangial region in IgAN patients, but its significance has not been clearly elucidated. It has been reported that the prevalence of IgM deposition in IgAN patients varies from $25.00-80.27 \%$ 9,18-22, these differences may be related to race, age, gender and the number of patients enrolled in the various studies. This multicenter, prospective, observational cohort of patients with biopsy-proven primary IgAN is the first to investigate the relationships between mesangial IgM deposition and renal disease progression in IgAN patients. Among a total of 1239 IgAN patients, mesangial IgM deposition was found in $42.45 \%$ of patients, with an average intensity of Grade 1 (33.15\%).

Glomerular IgM deposition was associated with proteinuria and unfavorable histopathological features, such as $\mathrm{M} 1, \mathrm{E} 1, \mathrm{C} 1 / \mathrm{C} 2$, and deposition of IgG, C3, C4 and C1q, independently of renal function decline (Tables 1 \& 2). In childhood IgA nephropathy, the presence of IgM and C1q correlates with heavy proteinuria at the time of renal biopsy ${ }^{8}$. However, a retrospective study from Turkey which enrolled 100 patients suggested no correlation between clinical parameters and positivity for $\operatorname{lgM}$ deposits ${ }^{9}$. The reason for the differences in these observations may be attributable in part to different indications for renal biopsy among countries, and different grades of renal injury among IgAN patients. 
In IgAN, the complement system may be activated in the glomeruli via the classical, alternative or mannose-binding lectin pathway ${ }^{3}$. IgG/IgM and C1q co-deposition may initiate the classical complement pathway through activation of $\mathrm{C} 2, \mathrm{C} 4$ and $\mathrm{C} 3$ to form the membrane attack complex (MAC), which eventually causes renal inflammation and fibrosis ${ }^{3}$. Thus, IgM forms the classic pathway convertase C4bC2a through C1 fixation and subsequently cleavage of C3 into C3a and C3b. Complement-activating moieties in the mesangial area result in additional C3 convertase formation, through the alternative pathway $\mathrm{C} 3 \mathrm{bBb}$. The alternative pathway $\mathrm{C} 3$ convertase subsequently cleaves $\mathrm{C} 3$ and generates more $\mathrm{C} 3$ cleavage products ${ }^{3}$. It was reported that the lectin pathway can also be triggered by $\operatorname{lgM}^{26}$. Therefore, we hypothesize that glomerular IgM, which binds to injury-associated epitopes, activates the complement system through the classic pathway and further amplifies it through the alternative or lectin pathway to induce prolonged and more severe type of glomerulonephritis. Consistent with this hypothesis, we observe correlation between the intensity of glomerular IgM deposits and the intensity of IgG, C3, C4 and C1q deposits (Table 1).

The PSM method was used in the current study to minimize bias between patients; meanwhile, this was a multicenter, prospective, observational study, which enhanced the accuracy and reliability of our results. Our data suggest that mesangial IgM deposition denotes a poor prognosis for patients with IgA nephropathy in terms of therapy responses and renal outcome. As shown in Table 3, the percent of patients reaching $\mathrm{CR}$ negatively correlates with the degree of glomerular IgM deposits; similarly, renal survival negatively correlates with higher degree of glomerular IgM deposits (Fig. 2). While COX multivariate analysis suggests that hypertension, urinary protein, eGFR, T1 or T2 lesion, IgM deposition are independent predictors of a combined endpoint (50\% reduction in eGFR and/or ESRD or death) in IgAN patients. Of note, and in variance with previous validation studies of Oxford classification, the present study reveals independent association of poor renal outcomes with $\mathrm{M}$ or $\mathrm{E}$ or $\mathrm{S}$ or $\mathrm{C}$ lesion ${ }^{23-25}$. Thus, the data are relevant because in addition to identifying a new prognostic factor, it supports the notion that activation of the complement system may play a crucial role in the pathogenesis of IgAN.

Although it's a multicenter, prospective, observational study, some study limitations should be noted as well. First, because of the observational design, it is difficult to determine whether prospective therapeutic intervention based on IgM deposition will affect the prognosis of IgAN patients. Second, all patients included in this study were from a single ethnicity (Han Chinese). Third, we did not measure serum IgM antibodies for correlation with mesangial IgM deposits. Finally, although we performed PS matching analysis to minimize bias related to age, gender, and treatment modality among patients with different degrees of IgM deposition, we could not exclude all confounding factors. Therefore, further research is needed for validation.

\section{Conclusions}

Different degrees of mesangial IgM deposition have a negative correlation with histological activity, clinical severity and renal outcome. Furthermore, mesangial IgM deposition is an independent risk factor 
for poor renal prognosis in IgAN. Thus, these findings suggest that glomerular IgM deposition is highly likely to play an important role in the progression of IgAN.

\section{Abbreviations}

IgAN: IgA nephropathy; ESRD: end stage renal disease; eGFR: estimated glomerular filtration rate; MAP: mean arterial pressure; PSM: propensity score matching

\section{Declarations}

\section{Acknowledgements}

The authors thank all of the participants and attending physicians for their contributions

\section{Authors' contributions}

Conception and design: LT, WQ, YT; Administrative support: WQ, YT; Collection and assembly of data LT, G-Q P, Z-X Z, J-X T, YM, D-G W, LZ; Data analysis and interpretation: LT; Manuscript writing: LT, DSH, WQ. All authors read and approved the final manuscript.

\section{Funding}

This study was partly supported by the Science and Technology Planning Project of Sichuan Province (No. 2019YFS0280).

\section{Availability of data and materials}

The datasets generated and/or analyzed during the current study are not publicly available due to privacy policy but are available from the corresponding author on reasonable request.

\section{Ethics approval and consent to participate}

This research was approved by the ethical committee of West China Hospital of Sichuan University, Affiliated Hospital of Zunyi Medical University, Zigong Third People's Hospital and People's Hospital of Mianzhu city. Written informed consent was obtained from each patient.

\section{Consent for publication}

Not applicable.

\section{Conflict of interest}

The authors declare no conflict of interest.

\section{References}


1. Wyatt RJ, Julian BA. IgA nephropathy. The New England journal of medicine. 2013;368(25):2402-14.

2. Moresco RN, Speeckaert MM, Delanghe JR. Diagnosis and monitoring of IgA nephropathy: the role of biomarkers as an alternative to renal biopsy. Autoimmunity reviews. 2015;14(10):847-53.

3. Berger SP, Roos A, Daha MR. Complement and the kidney: what the nephrologist needs to know in 2006? Nephrology, dialysis, transplantation: official publication of the European Dialysis and Transplant Association -. European Renal Association. 2005;20(12):2613-9.

4. Maillard N, Wyatt RJ, Julian BA, et al. Current Understanding of the Role of Complement in IgA Nephropathy. Journal of the American Society of Nephrology: JASN. 2015;26(7):1503-12.

5. Strassheim D, Renner B, Panzer S, et al. IgM contributes to glomerular injury in FSGS. Journal of the American Society of Nephrology: JASN. 2013;24(3):393-406.

6. Panzer SE, Laskowski J, Renner B, et al. IgM exacerbates glomerular disease progression in complement-induced glomerulopathy. Kidney international. 2015;88(3):528-37.

7. Platt JL, Cascalho M. IgM in the kidney: a multiple personality disorder. Kidney international. 2015;88(3):439-41.

8. Welch TR, McAdams J. Immunoglobulin M. and C1q mesangial labeling in IgA nephropathy. American journal of kidney diseases: the official journal of the National Kidney Foundation. 1998;32(4):589-92.

9. Heybeli C, Oktan MA, Yildiz S, et al. Clinical significance of mesangial IgM deposition in patients with IgA nephropathy. Clinical experimental nephrology. 2019;23(3):371-9.

10. Levey AS, Stevens LA. Estimating GFR using the CKD Epidemiology Collaboration (CKD-EPI) creatinine equation: more accurate GFR estimates, lower CKD prevalence estimates, and better risk predictions. American journal of kidney diseases: the official journal of the National Kidney Foundation. 2010;55(4):622-7.

11. Cattran DC, Coppo R, Cook HT, et al. The Oxford classification of IgA nephropathy: rationale, clinicopathological correlations, and classification. Kidney international. 2009;76(5):534-45.

12. Trimarchi H, Barratt J, Cattran DC, et al. Oxford Classification of IgA nephropathy 2016: an update from the IgA Nephropathy Classification Working Group. Kidney international. 2017;91(5):1014-21.

13. Tan L, Tang Y, Peng W, Mathew BS, Qin W. Combined Immunosuppressive Treatment May Improve Short-Term Renal Outcomes in Chinese Patients with Advanced IgA Nephropathy. Kidney blood pressure research. 2018;43(4):1333-43.

14. Little RJ, Rubin DB. Causal effects in clinical and epidemiological studies via potential outcomes: concepts and analytical approaches. Annual review of public health. 2000;21:121-45.

15. D'Agostino RB Jr. Propensity score methods for bias reduction in the comparison of a treatment to a non-randomized control group. Statistics in medicine. 1998;17(19):2265-81.

16. Nonparametric Preprocessing for Parametric Causal Inference Matchlt 
Ho D, Imai K, King G, Stuart EA. Matchlt: Nonparametric Preprocessing for Parametric Causal Inference.

17. Glassock RJ. The pathogenesis of IgA nephropathy. Current opinion in nephrology hypertension. 2011;20(2):153-60.

18. Hao Y, Zhao Y, Huang R, Fu P. Analysis of the relationship between Oxford classification, IgM deposition and multiple indexes and the adverse prognosis of patients with primary IgA nephropathy and related risk factors. Experimental therapeutic medicine. 2019;17(2):1234-9.

19. Zhu B, Zhu CF, Lin Y, et al. Clinical characteristics of IgA nephropathy associated with low complement 4 levels. Renal failure. 2015;37(3):424-32.

20. Nasri H, Sajjadieh S, Mardani S, et al. Correlation of immunostaining findings with demographic data and variables of Oxford classification in IgA nephropathy. Journal of nephropathology. 2013;2(3):190-5.

21. Shin DH, Lim BJ, Han IM, et al. Glomerular IgG deposition predicts renal outcome in patients with IgA nephropathy. Modern pathology: an official journal of the United States Canadian Academy of Pathology Inc. 2016;29(7):743-52.

22. Heybeli C, Unlu M, Yildiz S, Cavdar C, Sarioglu S, Camsari T. IgA nephropathy: association of C4d with clinical and histopathological findings and possible role of IgM. Renal failure. 2015;37(9):14649.

23. Coppo R, Troyanov S, Bellur S, et al. Validation of the Oxford classification of IgA nephropathy in cohorts with different presentations and treatments. Kidney international. 2014;86(4):828-36.

24. Coppo R. Corticosteroids in IgA Nephropathy: Lessons from Recent Studies. Journal of the American Society of Nephrology: JASN. 2017;28(1):25-33.

25. Sean J. Barbour Gabriela Espino-Hernandez HNR, Rosanna Coppo, lan SD, Roberts J, Feehally AM. Herzenberg and Daniel C. Cattran. The MEST score provides earlier risk prediction in IgA nephropathy.pdf. Kidney international. 2016;89:167-75.

26. McMullen ME, Hart ML, Walsh MC, Buras J, Takahashi K, Stahl GL. Mannose-binding lectin binds IgM to activate the lectin complement pathway in vitro and in vivo. Immunobiology. 2006;211(10):759-66.

\section{Tables}

Due to technical limitations, Tables 1-5 are only available as a download in the supplemental files section

\section{Figures}




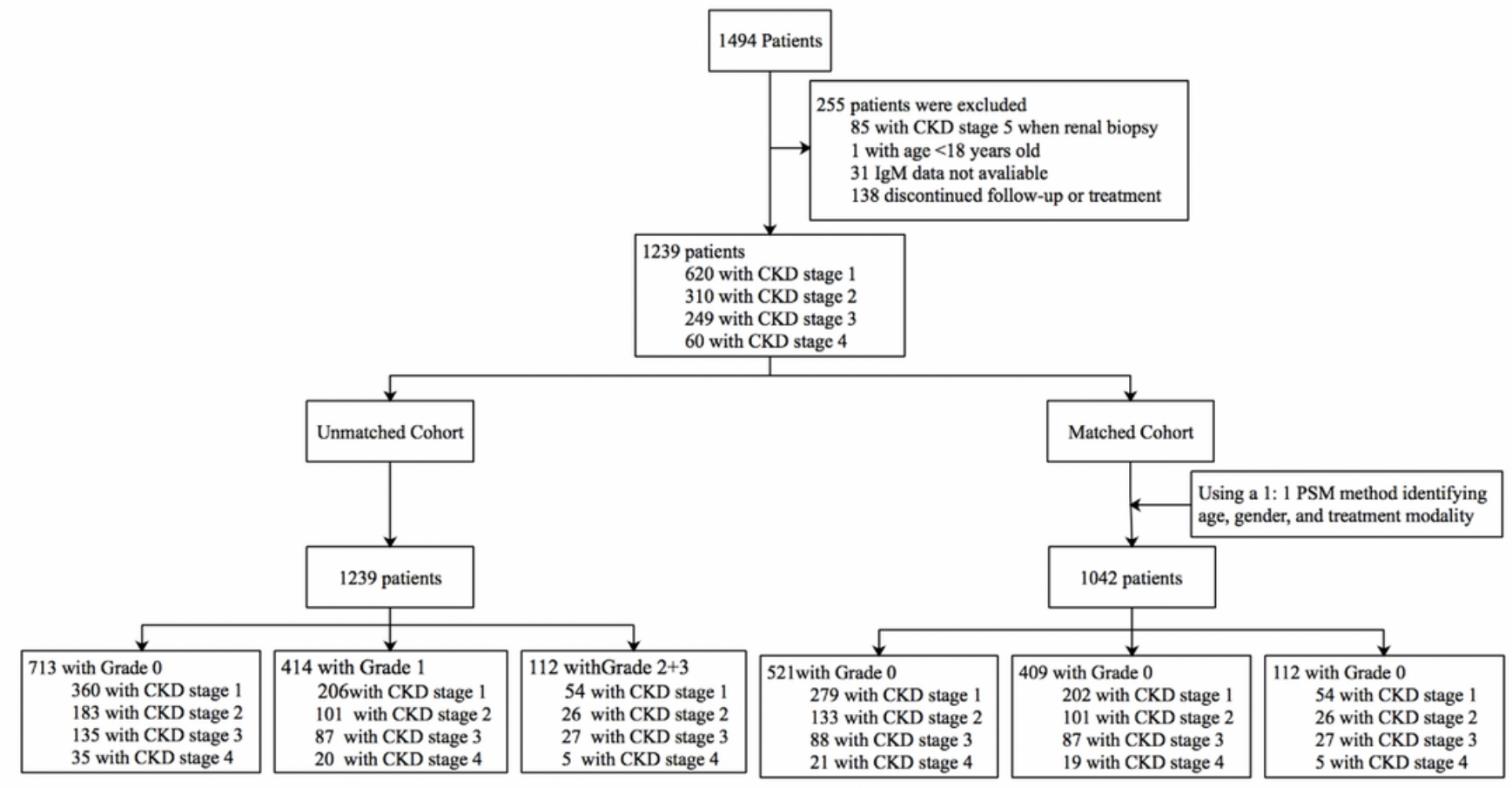

Abbreviations: CKD, chronic kidney disease; PSM, propensity score matching

\section{Figure 1}

Study profile.
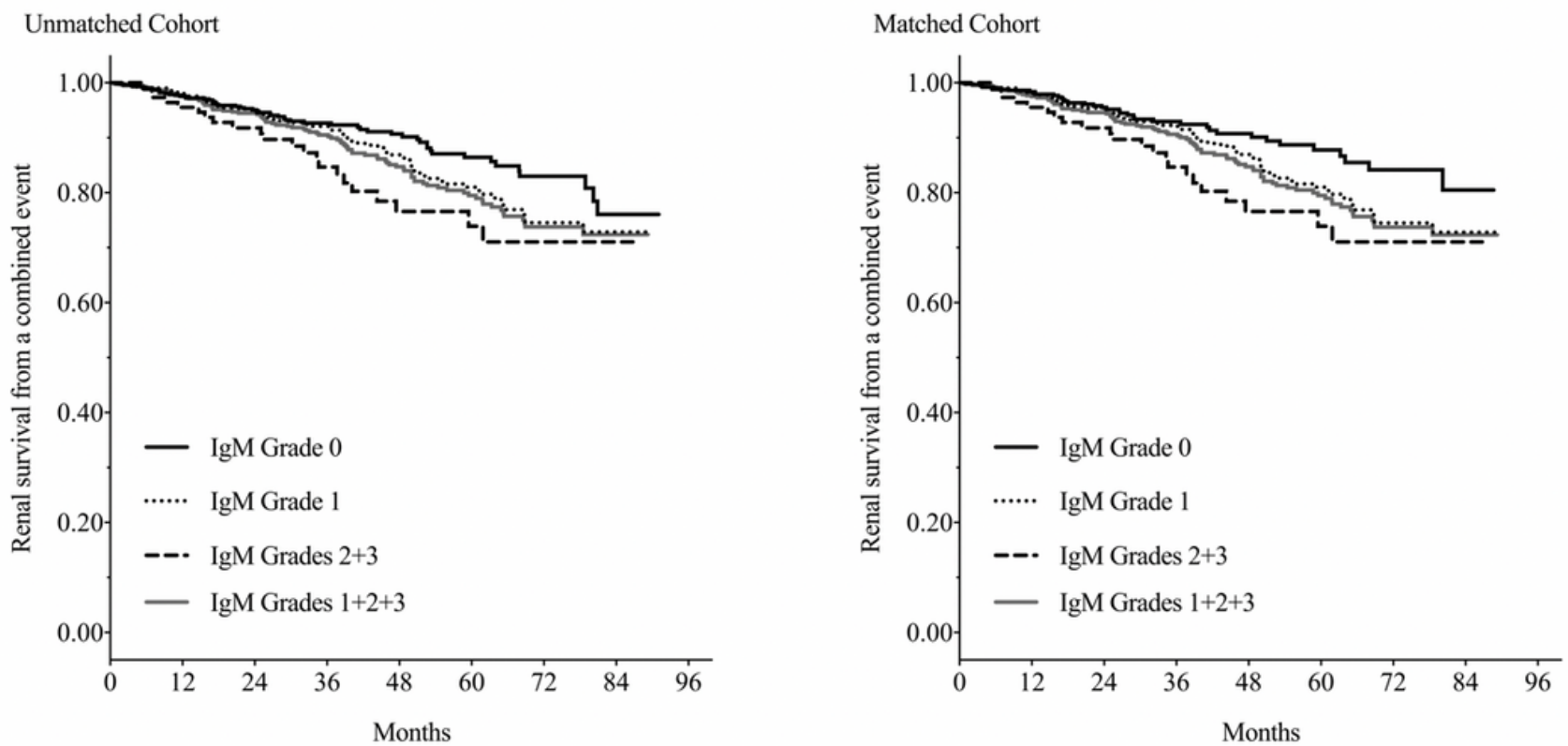

Abbreviations: Grade 0, no or trace IgM deposits; Grade 1, mild IgM deposits; Grade 2, moderate IgM deposits; Grade 3, marked IgM deposits.

Figure 2

Kaplan-Meier analysis for the probability of composite endpoint. 


\section{Supplementary Files}

This is a list of supplementary files associated with this preprint. Click to download.

- Table5.pdf

- Table2.pdf

- Table3.pdf

- Table4.pdf

- Table1.pdf 